\begin{tabular}{|c|l|}
\hline Title & $\begin{array}{l}\text { Nonreentrant atrioventricular nodal tachycardia due to triple nodal pathways manifested by radiofrequency ablation at } \\
\text { coronary sinus ostium }\end{array}$ \\
\hline Author(s) & Yokoshiki, Hisashi; Sasaki, Koji; Shimokawa, Junichi; Sakurai, Masay uki; Tsutsui, Hiroyuki \\
\hline Citation & $\begin{array}{l}\text { Journal of Electrocardiology, 39(4), 395-399 } \\
\text { https://doi.org/40.1016/.jelectrocard.2005.08.017 }\end{array}$ \\
\hline Issue Date & $2006-10$ \\
\hline Doc URL & http:/hdl.handle.net/2115/15828 \\
\hline Type & article (author version) \\
\hline File Information & JE39-4.pdf \\
\hline
\end{tabular}

Instructions for use 


\section{Nonreentrant atrioventricular nodal tachycardia due to triple nodal pathways manifested by radiofrequency ablation at coronary sinus ostium}

Hisashi Yokoshiki, MD, $\mathrm{PhD}^{\mathrm{a}}$, Koji Sasaki, MD, $\mathrm{PhD}^{\mathrm{a}}$, Junichi Shimokawa, $\mathrm{MD}^{\mathrm{a}}$, Masayuki Sakurai, MD, $\mathrm{PhD}^{\mathrm{b}}$, Hiroyuki Tsutsui, $\mathrm{MD}, \mathrm{PhD}^{\mathrm{a}}$

${ }^{a}$ Department of Cardiovascular Medicine, Hokkaido University Graduate School of Medicine, Sapporo, Japan

${ }^{\mathrm{b}}$ Department of Cardiovascular Medicine, Hokko Memorial Hospital, Sapporo, Japan

Short title: Nonreentrant AVN tachycardia by RF delivery

Address for correspondence: Hisashi Yokoshiki MD, PhD

Department of Cardiovascular Medicine, Hokkaido University Graduate School of Medicine, Kita-15, Nishi-7, Kita-ku, Sapporo 060-8638, Japan.

Phone: 81-11-706-6973

Fax: 81-11-706-7874

E-mail: yokoshh@med.hokudai.ac.jp 


\begin{abstract}
We report a case of complex supraventricular tachycardia manifested by radiofrequency delivery. Initially, the patient presented with orthodromic AV reentrant tachycardia via a left-sided accessory pathway that was successfully eliminated. Atrial tachycardia originating from coronary sinus ostium was also induced. Radiofrequency delivery at the coronary sinus ostium induced a narrow QRS tachycardia with irregular RR intervals. A detailed analysis explained that the tachycardia could be a nonreentrant mechanism due to triple AV nodal pathways: an atrial excitation evokes double ventricular response due to simultaneous activation of the fast and slow pathways, and the next one activates ventricle through the intermediate pathway.
\end{abstract}

Key Words: triple nodal pathways, nonreentrant atrioventricular nodal tachycardia, double ventricular response, coronary sinus ostium, radiofrequency ablation 


\section{Introduction}

Since Wu et al. reported double ventricular response (DVR) due to simultaneous activation of dual atrioventricular (AV) nodal pathways in 1975, there have been several case reports of the nonreentrant tachycardia related to DVR [1 - 12]. However, to the best of our knowledge, there is only one case report by Arena et al. [10] describing the nonreentrant tachycardia due to triple nodal pathways. In the present case report, this unusual nonreentrant tachycardia due to multiple nodal pathways was induced by radiofrequency (RF) energy delivery for elimination of the atrial tachycardia originating from coronary sinus (CS) ostium.

\section{Case Report}

A 40-year-old woman was admitted to our hospital for frequent episodes of paroxysmal palpitations. The 12-lead ECG during sinus rhythm showed pre-excitation syndrome (i.e., Wolff-Parkinson-White syndrome) whose QRS complex at the right precordial leads was predominantly upright, suggesting presence of a left-sided accessory pathway. No evidence of structural heart disease was found by echocardiography.

After obtaining written informed consent, the electrophysiological study was performed for evaluating the mechanism of her palpitation and its treatment. Under local anesthesia with mild conscious sedation, three quadripolar catheters were inserted from the right femoral vein and positioned at the high right atrium (HRA), His-bundle region (His), and right ventricular apex (RVA). A decapolar catheter (2-8-2 mm interelectrode spacing) was inserted from the left subclavian vein and positioned in the coronary sinus (CS), with the proximal electrode located close to the CS ostium. 
Rapid pacing (S1S1 = 300) at RVA induced a narrow QRS tachycardia as shown in Fig. 1A. The earliest atrial activation was detected in CS 4-5, indicating that the tachycardia was orthodromic AVRT via a left-lateral accessory pathway. On the other hand, after a drive cycle length of 600 msec (S1S1 $=600$ ), a single extrastimulus of 260 msec (S1S2 = 260) at HRA induced a wide QRS tachycardia whose configuration resembled her pre-excited ECG (Fig. 1B). An atrial echo beat initiated this tachycardia, with the sequence of A-A-V pattern. During the tachycardia, the earliest atrial activation site was near the CS ostium (Fig. 1B). In addition, after the successful radiofrequency (RF) ablation of the left-sided accessory pathway, the similar tachycardia originated from the vicinity of CS ostium was induced by an extrastimulus of $220 \mathrm{msec}$ (S1S1/S1S2 = 500/220) at HRA in presence of isoproterenol (Fig. 1C). The AV conduction during the tachycardia sometimes exhibited the Wenchebach periodicity (Fig. 1C). Therefore, the tachycardia given in Fig. 1B and Fig. 1C was considered to be atrial tachycardia (AT) originating from CS ostium. However, we cannot completely negate the possibility that it is AV nodal reentrant tachycardia with Wenchebach AV block.

The property of AV conduction after the ablation of accessory pathway was summarized in Fig. 2. As shown in Fig. 2A, the ERP of antegrade AV nodal conduction was 290 ms without jump up of AH interval. During the pacing at RVA, the VA conduction with the earliest atrial activation of CS ostium was occasionally observed (Fig. 2B). This VA conduction was very long and exhibited the decremental property (Fig. 2C). These results indicated the presence of slow pathway with weak retrograde conduction.

In order to eliminate the AT, RF energy with the target temperature of $50{ }^{\circ} \mathrm{C}$ was applied at CS ostium. A few seconds later, AV junctional rhythm emerged with AV 
dissociation (Fig. 3A), which promptly led us to stop the RF delivery. At the same time, a narrow QRS tachycardia of irregular RR intervals was initiated by an atrial premature contraction originating nearby His bundle (Fig. 3A) and was sustained (Fig. 3B). As given in the laddergrams in Figs $3 \mathrm{~A}$ and $3 \mathrm{~B}$, this tachycardia could be explained by a nonreentrant mechanism due to triple AV nodal pathways. That is, an atrial excitation evokes ventricular activation twice via the fast and slow AV nodal pathways (double ventricular response: DVR), and the next one activates the ventricle through the intermediate AV nodal pathway. An atrial overdrive pacing terminated the tachycardia. The AT from CS ostium was no longer induced even in presence of isoproterenol.

\section{Discussion}

It is essential for the occurrence of DVR to a single atrial depolarization that time difference between AV nodal fast and slow pathway conduction is longer than the refractory period of His-Purkinje system. In general, the slow pathway conduction time is markedly prolonged. It was reported that VA conduction was weak or absent in patients with DVR [4, 6, 8]. Lin et al. [6] speculated that blockade of the retrograde slow pathway conduction via fast pathway activation is required for DVR. Consistently, in the present case, AV conduction time through the slow pathway was prolonged during the tachycardia (Figs 3A and 3B) and the VA conduction presumably via the slow pathway was only intermittently observed (Fig. 2).

On the other hand, the novelty of this report is that RF application at CS ostium induced a sustained nonreentrant tachycardia due to triple AV nodal pathways: simultaneous fast and slow pathway conduction in response to a single atrial 
depolarization followed by intermediate pathway conduction. As far as we know, the tachycardia similar to the present case was only reported by Arena et al. [10]. The present case may help elucidate the mechanism of this tachycardia, because its onset was observed in response to RF energy application.

The proposed mechanism of the tachycardia is shown in Fig. 4. Under the control condition, there would be the retrograde concealed conduction (indicated as thick curved arrow) of the slow pathway after the fast pathway excitation, thereby preventing DVR (Fig. 4A). In contrast, this concealed conduction could be abolished or attenuated by RF application at CS ostium. This allows simultaneous antegrade conduction of dual (i.e., fast and slow) AV nodal pathways (Fig. 4B). Due to the refractoriness of His-Purkinje system produced by the slow pathway conduction, the next atrial excitation would propagate through the intermediate pathway.

To the best of our knowledge, this is the first report to demonstrate the sustained nonreentrant tachycardia due to triple nodal pathways that was induced by RF energy application at CS ostium. The retrograde concealed conduction of slow pathway might be attenuated by RF delivery, thereby inducing this unusual tachycardia. 


\section{References}

1. Wu D, Denes P, Dhingra R, Pietras RJ, Rosen KM. New manifestations of dual A-V nodal pathways. Eur J Cardiol. 1975; 2: 459-466.

2. Csapo G. Paroxysmal nonreentrant tachycardias due to simultaneous conduction in dual atrioventricular nodal pathways. Am J Cardiol. 1979; 43: 1033-1045.

3. Gomes JA, Kang PS, Kelen G, Khan R, El-Sherif N. Simultaneous anterograde fastslow atrioventricular nodal pathway conduction after procainamide. Am J Cardiol. 1980; 46: 677-684.

4. Sutton FJ, Lee YC. Supraventricular nonreentrant tachycardia due to simultaneous conduction through dual atrioventricular nodal pathways. Am J Cardiol. 1983; 51: 897-900.

5. Sutton FJ, Lee YC. Paroxysmal nonreentrant tachycardia due to simultaneous conduction via dual atrioventricular nodal pathways. Am Heart J. 1985; 109: 157-159.

6. Lin FC, Yeh SJ, Wu D. Determinants of simultaneous fast and slow pathway conduction in patients with dual atrioventricular nodal pathways. Am Heart J. 1985; 109: 963-970.

7. Matsuhisa M, Shimomura K, Ohe T, Kamakura S, Aihara N. Double atrial and double ventricular responses during slow-fast fast-slow atrioventricular nodal reentrant tachycardia. Pacing Clin Electrophysiol. 1989; 12: 1381-1386.

8. Sakurada H, Sakamoto M, Hiyoshi Y, Tejima T, Motomiya T, Sugiura M, Hiraoka M. Double ventricular responses to a single atrial depolarization in a patient with dual AV nodal pathways. Pacing Clin Electrophysiol. 1992; 15: 28-33. 
9. Ajiki K, Murakawa Y, Yamashita T, Oikawa N, Sezaki K, Kotsuka Y, Furuse A, Omata M. Nonreentrant supraventricular tachycardia due to double ventricular response via dual atrioventricular nodal pathways. J Electrocardiol. 1996; 29: 155160.

10. Arena G, Bongiorni MG, Soldati E, Gherarducci G, Mariani M. Incessant nonreentrant atrioventricular nodal tachycardia due to multiple nodal pathways treated by radiofrequency ablation of the slow pathways. J Cardiovasc Electrophysiol. 1999; 10: 1636-1642.

11. Kirchhof P, Loh P, Ribbing M, Wasmer K. Incessant supraventricular tachycardia with constant 1:2 atrioventricular ratio: a longitudinally dissociated atrioventricular node? J Cardiovasc Electrophysiol. 2003; 14: 316-319.

12. Mansour M, Marrouche N, Ruskin J, Natale A, Keane D. Incessant nonreentrant tachycardia due to simultaneous conduction over dual atrioventricular nodal pathways mimicking atrial fibrillation in patients referred for pulmonary vein isolation. $\mathrm{J}$ Cardiovasc Electrophysiol. 2003; 14: 752-755. 


\section{Figure legends}

Figure 1. Two types of tachycardia induced during the electrophysiological study.

Shown are the surface ECGs, leads I, II, and V1, and intracardiac electrograms recorded from high right atrium (HRA), His-bundle region (His d and His p), distal (CS d) to proximal (CS p) coronary sinus and right ventricular apex (RVA d). The position of CS p was close to the ostium of coronary sinus. (However, the His bundle electrograms are not well seen in the following examples.)

A: Rapid pacing with a cycle length of $300 \mathrm{msec}$ at right ventricular apex induced a narrow QRS tachycardia whose earliest atrial activation was observed at CS 4-5, i.e., left lateral position. B: Premature atrial stimulation at an S1-S2 interval of 260 msec after basic drives (S1-S1) of 600 msec induced a wide QRS tachycardia whose morphology was identical to preexcited QRS complex. The earliest atrial activation was observed at CS p that was located around the coronary sinus ostium. C: After successful radiofrequency ablation of the left-sided accessory pathway, a narrow QRS tachycardia, whose atrial activation sequence was similar to that in panel B, was induced by premature atrial stimulation (in presence of isoproterenol). AV block with a clear Wenchebach periodicity occurred during the tachycardia.

Figure 2. Anterograde and retrograde AV nodal conduction after radiofrequency ablation of the left-sided accessory pathway.

A: Anterograde AV nodal conduction curve. No apparent discontinuity (i.e., $50 \mathrm{msec}$ and more increase of $\mathrm{H} 1 \mathrm{H} 2$ intervals) of the curve was seen. B: Pacing at cycle length of 600 msec from RVA. There were intermittent ventriculoatrial (VA) conductions whose atrial 
activation was earliest at the ostium of coronary sinus (CS p). C: The VA conduction time was long (ca. $300 \mathrm{msec}$ ) and exhibited the decremental property.

Figure 3. Induction of a narrow QRS tachycardia with irregular RR intevals by atrail premature contraction during radiofrequency application.

A: During radiofrequency energy delivery at the coronary sinus ostium, atrial premature contraction originated around His bundle region induced a narrow QRS tachycardia with irregular RR intervals. Note that it was preceded by AV junctional rhythm with AV dissociation. The laddergram explains the proposed mechanism of the tachycardia. The fast, intermediate and slow pathways are given as solid ( - ), dash $\left({ }^{---^{-}}\right)$and dotted ( - - ) lines, respectively. Dotted areas represent refractory periods of the distal conducting system. B: As shown in the laddergram, it was suggested that an atrial excitation evoked ventricular activation twice via the fast and slow AV nodal pathways (double ventricular response: DVR) and the next one activated the ventricle through the intermediate AV nodal pathway. (The His bundle electrograms are not clear in this example.)

A: atrium; AVN: atrioventricular node; V: ventricle.

Figure 4. The proposed mechanism of the tachycardia induced by radiofrequency energy application at the coronary sinus ostium.

A: Control condition. B: Radiofrequency energy application.

FP: fast pathway; IP: intermediate pathway; SP: slow pathway; AVN: compact AV node; CS: coronary sinus.

The retrograde concealed conduction of the slow pathway via the fast pathway excitation is indicated as thick curved arrow. See text for detail. 
A

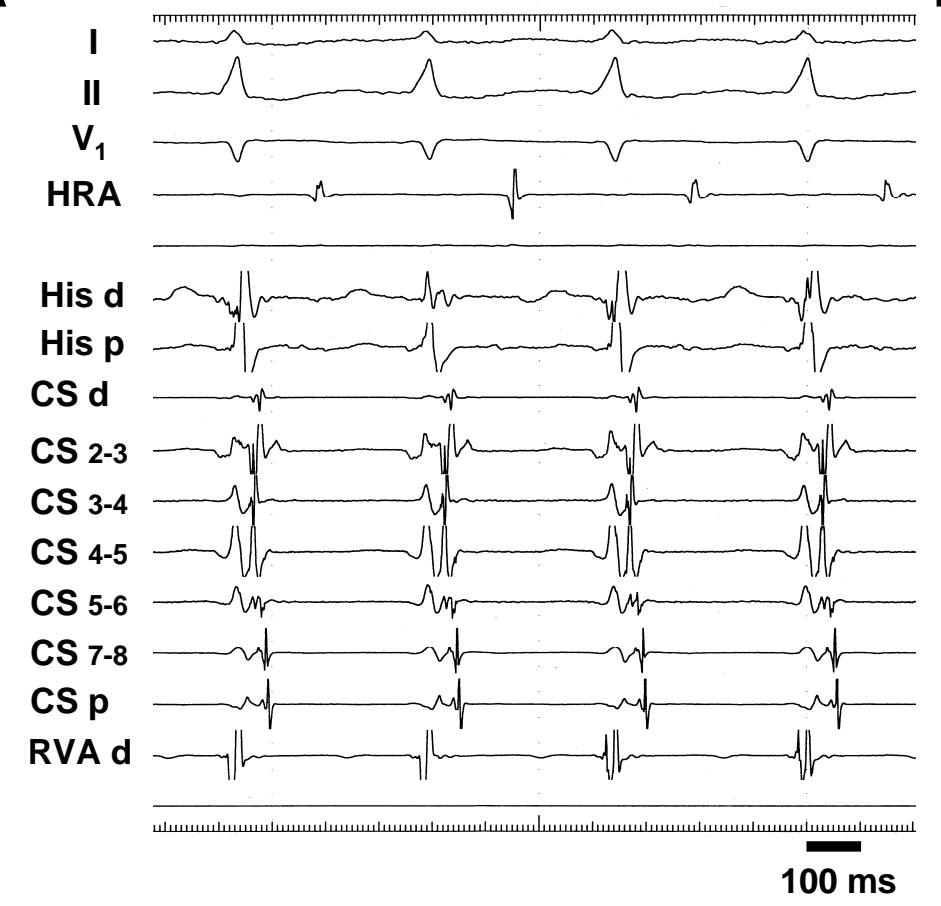

B

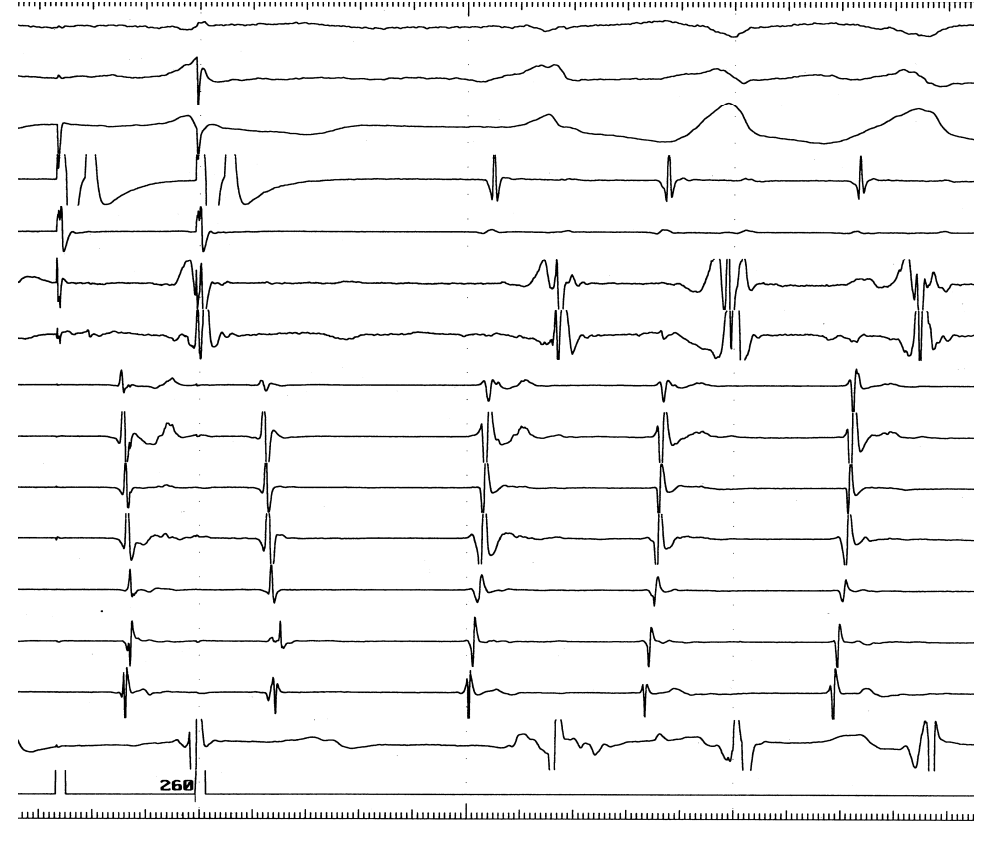

\section{$100 \mathrm{~ms}$}

C

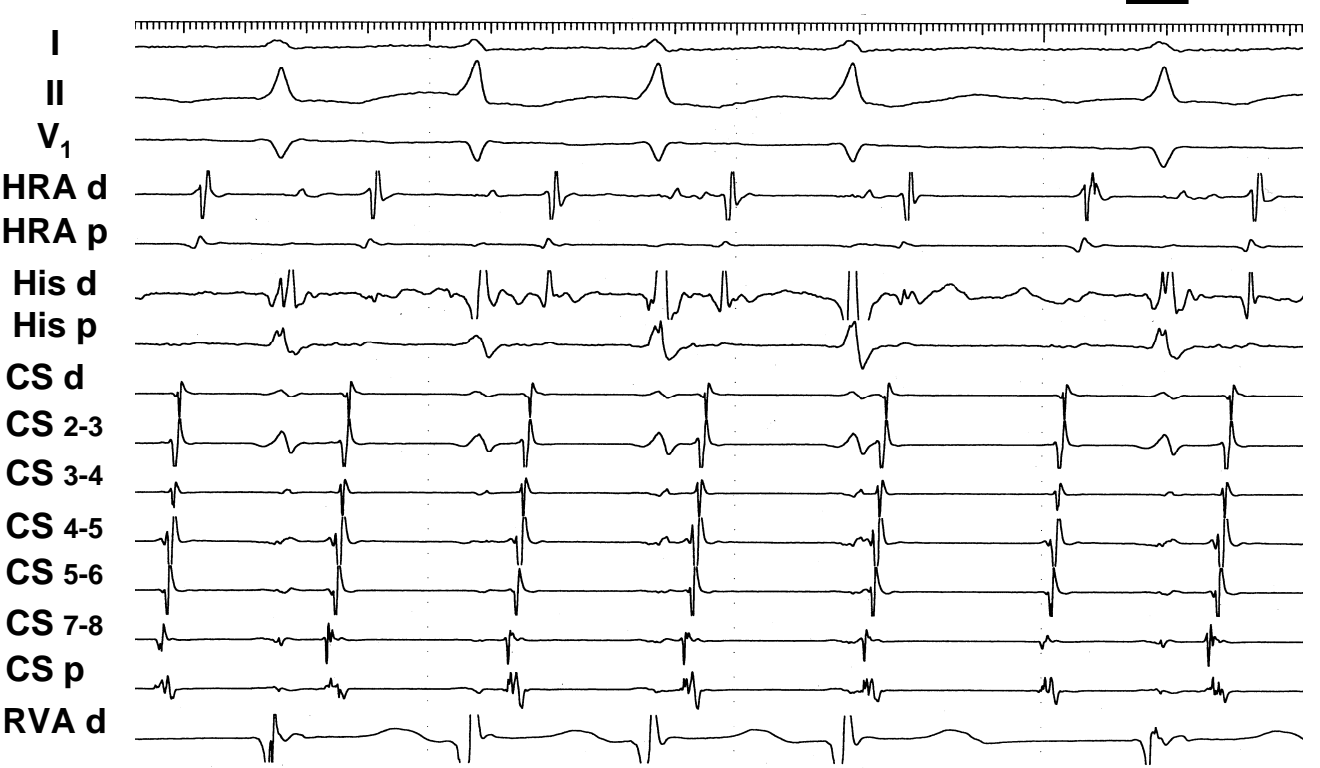

Fig. 1 
A

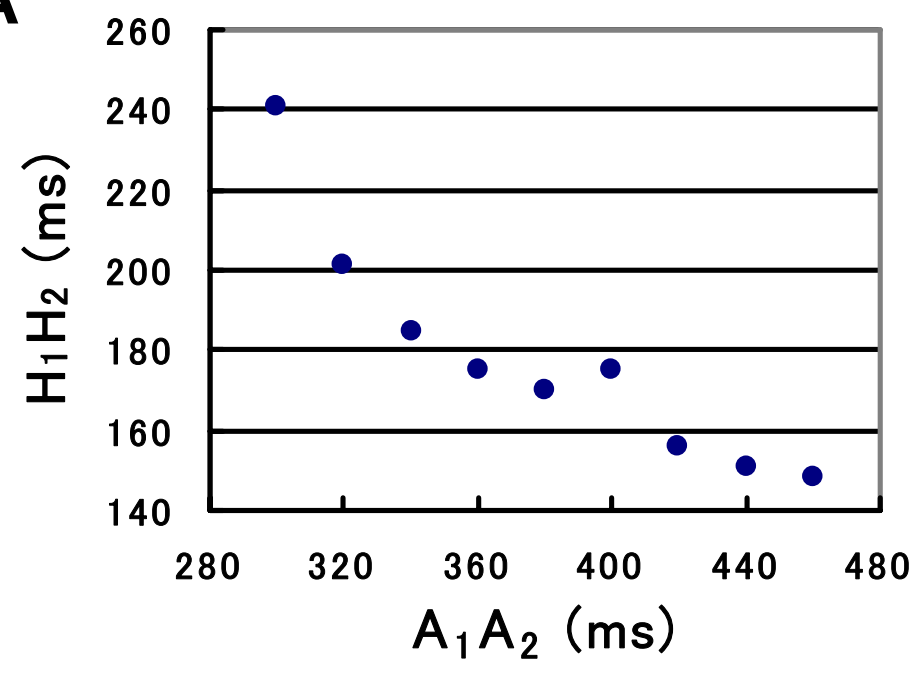

C

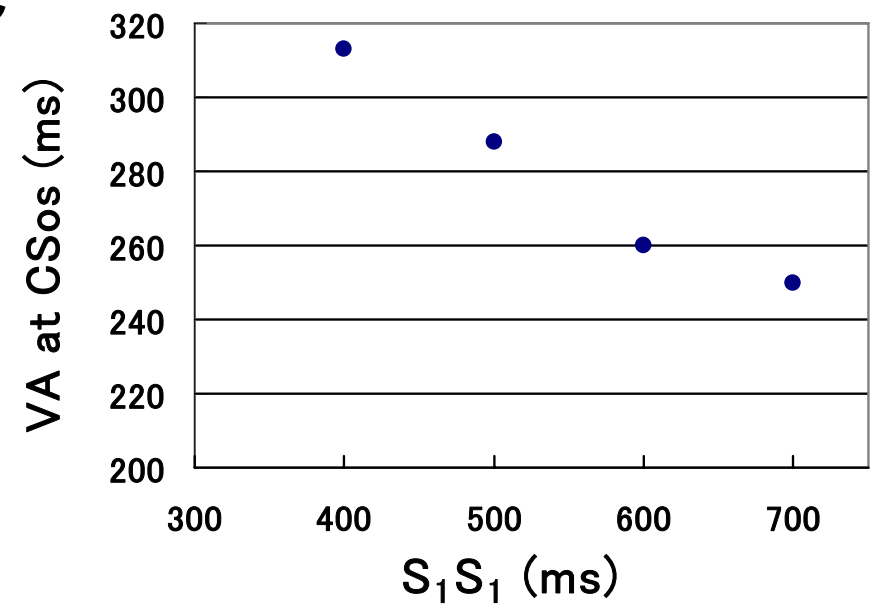

B

$200 \mathrm{~ms}$

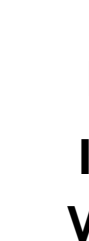

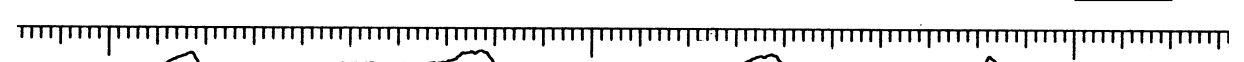
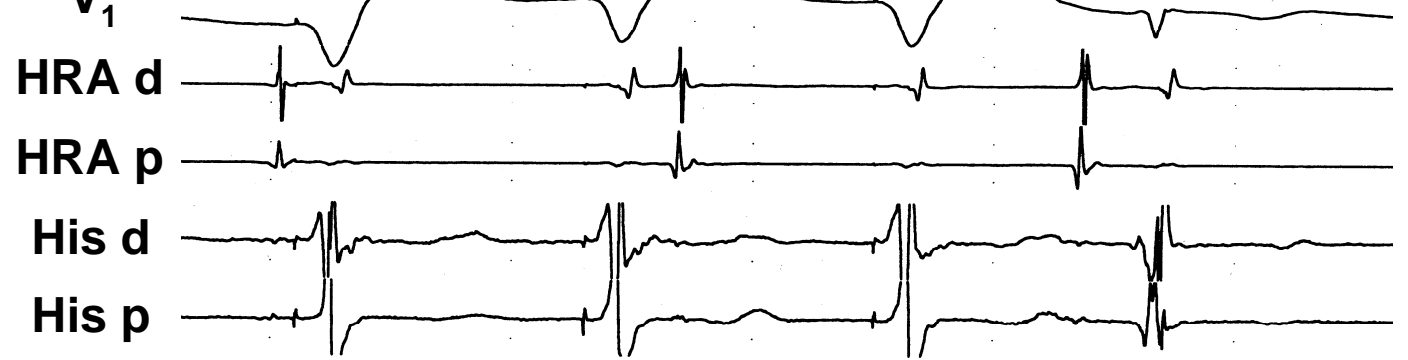

CS d

CS 2-3

CS 3-4

CS 4-5

CS 5-6

CS 7-8

CS $p$

RVA d
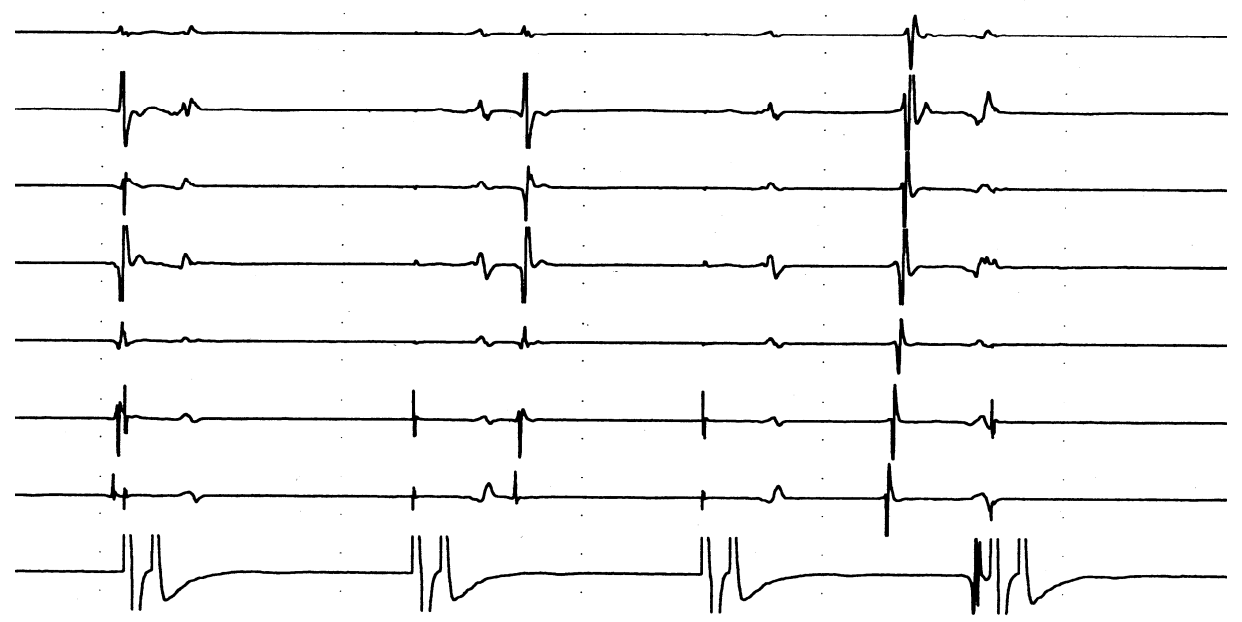

Fig. 2 
A

Control

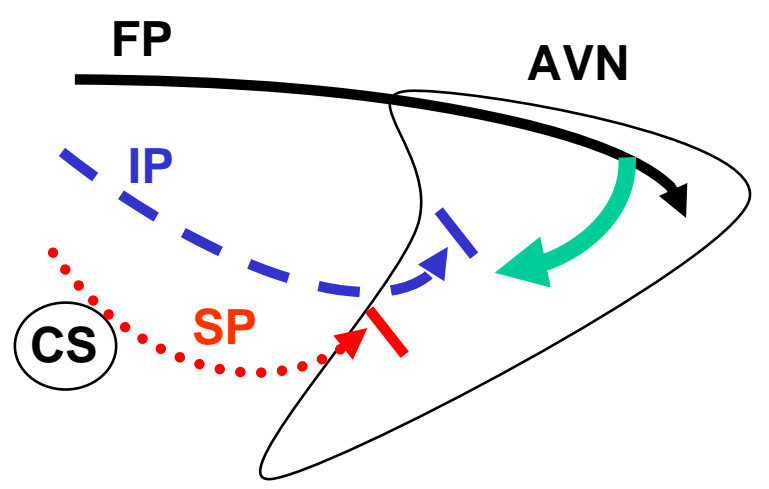

B RF application

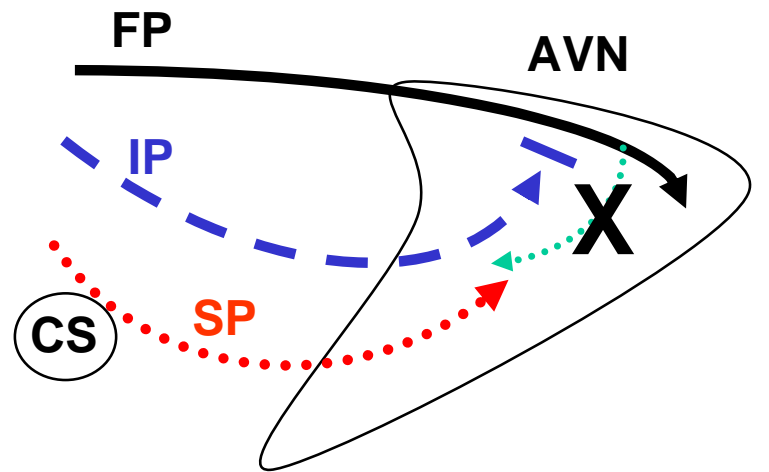

Fig. 4 

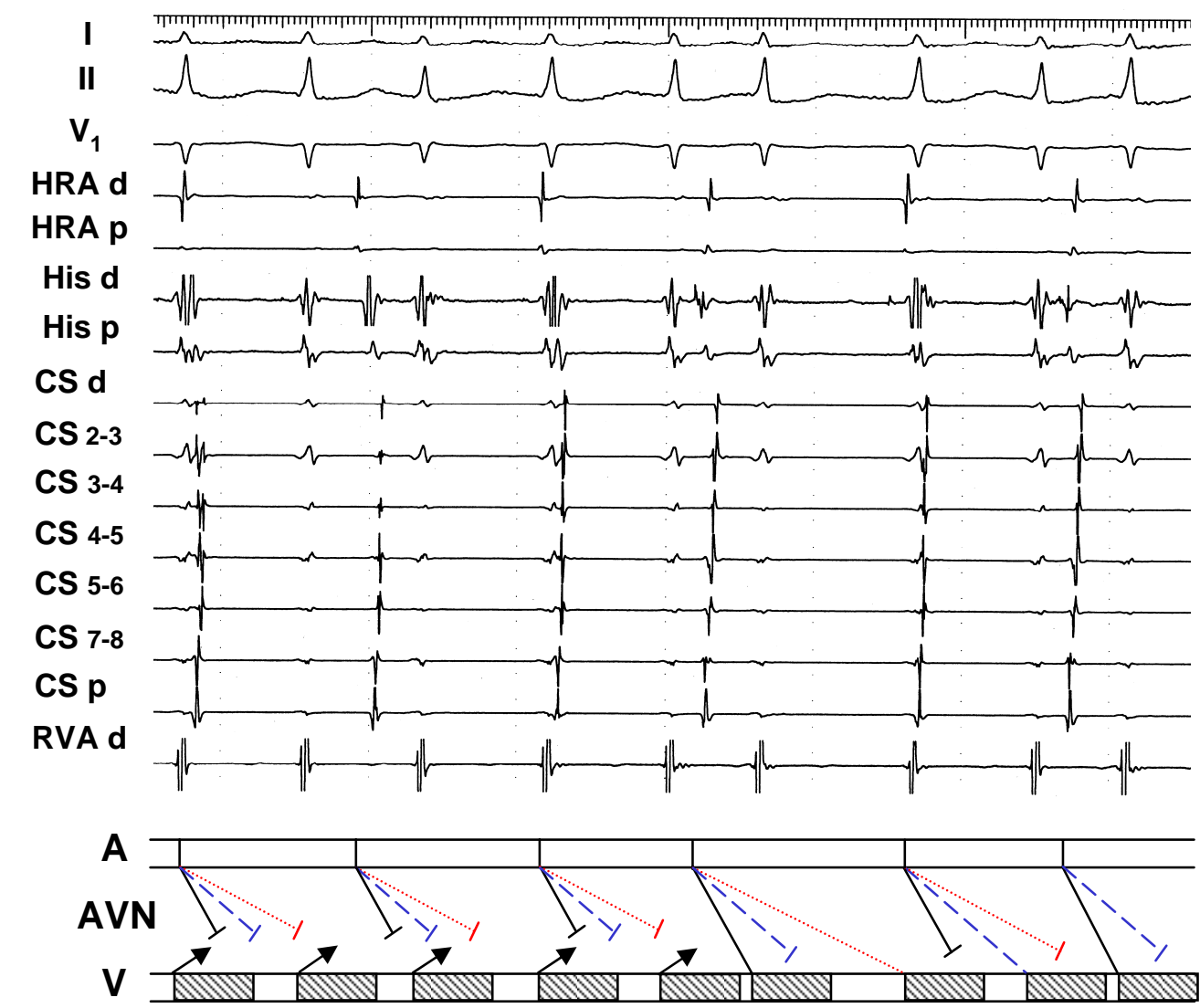

B

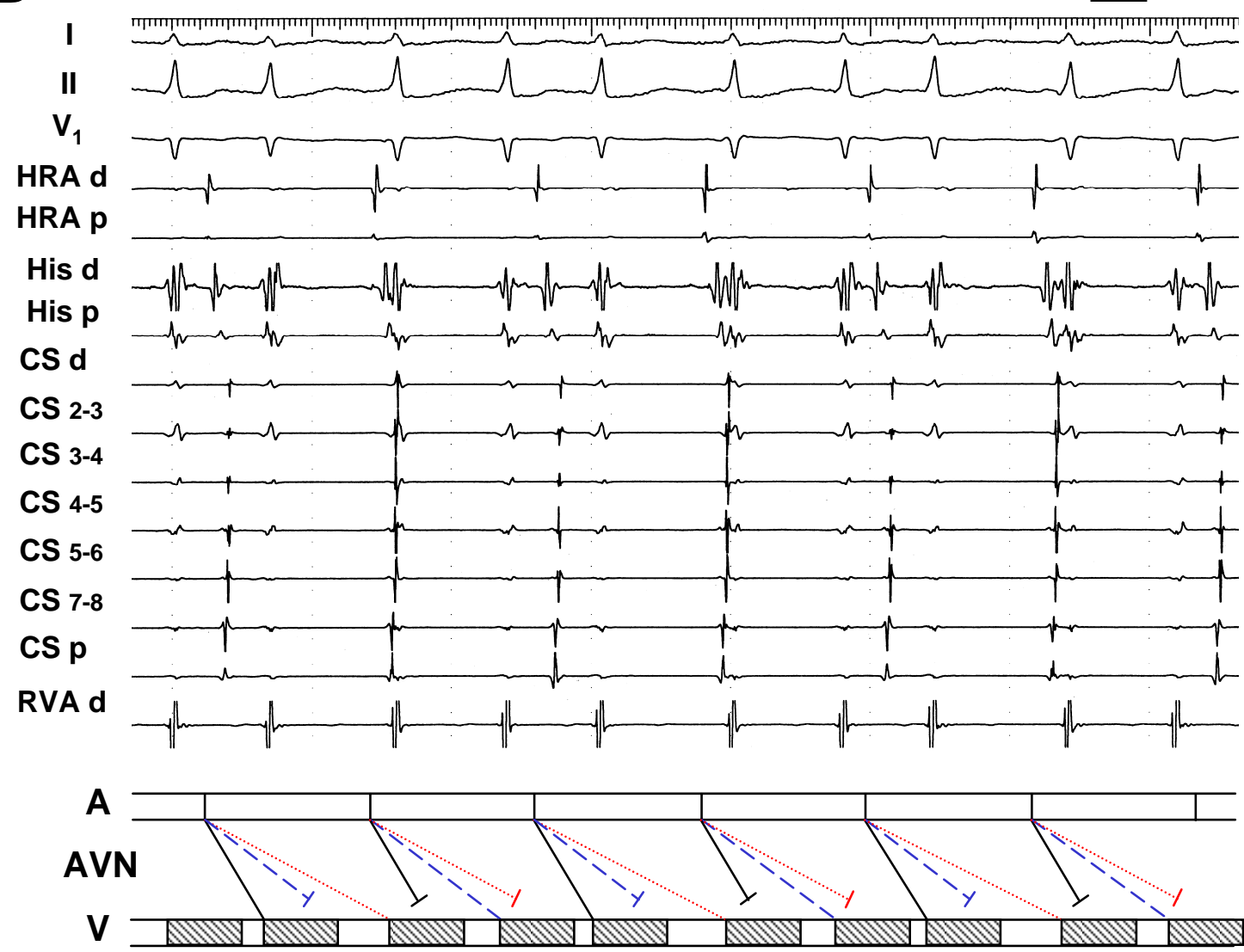

